Proc Indian Natn Sci Acad $\mathbf{8 1}$ No. 1 February 2015 Special Issue, pp. 56-61

(C) Printed in India.

DOI: $10.16943 /$ ptinsa/2015/v81i1/48051

\author{
Study of Fluctuations from Polyakov-Nambu-Jona-Lasinio Model \\ A BHATTACHARYYA ${ }^{1}, \mathrm{~S}_{\mathrm{DAS}}{ }^{2,3}, \mathrm{~S} \mathrm{~K} \mathrm{GHOSH}^{2,3}, \mathrm{~S} \mathrm{RAHA}^{2,3}, \mathrm{R} \mathrm{RAY}^{2,3}, \mathrm{~K}$ \\ SAHA $^{2,3}$ and S UPADHAYA ${ }^{* 2,3}$ \\ ${ }^{1}$ Department of Physics, University of Calcutta, 92, APC Road, Kolkata 700 009, India \\ ${ }^{2}$ Bose Institute, Center for Astroparticle Physics and Space Science Kolkata 700 091, India \\ ${ }^{3}$ Department of Physics, Bose Institute, 93/1, APC Road, Kolkata 700 009, India
}

(Received on 30 April 2014; Accepted on 9 September 2014)

\begin{abstract}
We compute various orders of fluctuations of conserved charges like baryon number and charge, which are of utmost importance in the quest for the dynamical properties of the exotic matter produced in high energy heavy ion collision experiments, viz. Quark-Gluon Plasma (QGP). Alongside analysis of their behavior with temperature and chemical potentials, the study is realized under the spectrum of contrasting experimental conditions as well.
\end{abstract}

Key Words : Quark-Gluon Plasma; Susceptibility; Fluctuation; PNJL Model

\title{
Introduction
}

Strongly interacting matter at non-zero baryon density and high temperature has become the cynosure of researchers all over the world. The exotic state of matter produced in such extreme conditions provide distinctive opportunities to theorists and experimentalists to deal with a downright new state. This state is the deconfined state of matter, comprising of elementary constituents, quarks and gluons and hence called Quark-Gluon Plasma (QGP) (Meyer-Ortmanns H, 1996). For the characterization of such a system, fluctuations and correlations (Koch V, 2008; Jeon S, 2004) play a pivotal role. They provide a measure of the susceptibilities of the system which further govern the response of the system to external forces. On the other hand, study of susceptibilities are of utmost importance in the context of heavy-ion physics since experimentally measured event-by-event fluctuations are associated to them. Their behavior in the phase transition regime carries important information regarding physics at finite chemical potential as well (Gavai

*Author for Correspondence : E-mail: sudipa@jcbose.ac.in 
et al., 2001). Most importantly, susceptibilities of a system are related to the moments of conserved quantities which serve as viable signals in the search for transition properties in strongly interacting systems. As for example, net charge fluctuations studied in terms of net charge susceptibilities, quantified by the $D$ - measure (Bhattacharyya et al., 2012) provide useful information regarding production of QGP.

Our present work delves in the study of quark number susceptibilities for both zero and non-zero quark chemical potential, $\mu_{q}$. We are also on the lookout for an insight into the behavioral nature of various orders of baryon number and charge susceptibilities under different experimental conditions. The whole work has been done under the framework of Polyakov Nambu-Jona-Lasinio model with 2-flavor consideration.

\section{PNJL Model}

Generally the QCD inspired phenomenological models are easier to handle compared to Lattice. But in all these models despite simplicity, the absence of proper order parameter for deconfinement transition gives rise to various uncertainties in their study, thereby reducing their predictive power. The thermal average of the Polyakov Loop can be considered to be the order parameter for deconfinement transition. Thus judiciously using Polyakov Loop in effective models is advantageous. The motivation behind the PNJL model is that here the chiral and deconfinement order parameters are entwined into a single framework. The details of the model used for 2 and 2+1 flavor systems may be found in (Ratti et al., 2006; Ghosh et al., 2008; Bhattacharyya et al., 2010a, Bhattacharyya et al., b, Bhattacharyya et al., 2011, Bhattacharyya et al., 2012a).

\section{Formalism}

Given the thermodynamic potential $\Omega$ in the PNJL model, we first solve numerically for the fields $\sigma, \phi$ and

$\bar{\phi}$ by proper minimization of the former. Field values thus obtained are then put back into $\Omega$ to obtain corresponding pressure, which can then be expanded in taylor series with the coefficients as susceptibilities. We find an effective measure for the fluctuations inherent in our model by suitably characterizing the system's susceptibilities defined as :

$$
\chi_{B}^{(n)}\left(\frac{T}{T_{c}}, \frac{\mu_{B}}{T}\right)=\frac{\partial^{n}}{\partial\left(\frac{\mu_{B}}{T}\right)^{n}}\left(\frac{P\left(\frac{T}{T_{c}}, \frac{\mu_{B}}{T}\right)}{T^{4}}\right)
$$

Similarly, we can define $\chi_{Q}^{(n)}$. Now these diagonal susceptibilities lead to fluctuations for grandcanonical ensemble in thermodynamic equilibrium. The $\mathrm{n}$-th cumulant of baryon number fluctuation $\left[B^{n}\right]$ is defined as

$$
\left[B^{n}\right]=V T^{3} \chi_{B}^{(n)}\left(\frac{T}{T_{c}}, \frac{\mu_{B}}{T}\right)
$$


The cumulants were reported as the variance $\sigma^{2}=\left[B^{2}\right]$, skewness $S=\frac{\left[B^{3}\right]}{\left[B^{2}\right]^{3 / 2}}$ and the kurtosis $\kappa=\frac{\left[B^{4}\right]}{\left[B^{2}\right]^{2}}$. The presence of volume $\mathrm{V}$ in eqn. (2), makes it paralyzed for direct use in the context of heavy ion experiments. So we rather opt for suitable ratios of them (Gupta et al., 2011):

$$
\begin{gathered}
m_{1}=S \sigma=\frac{\left[B^{3}\right]}{\left[B^{2}\right]}=\frac{\chi_{B}^{(3)}}{\chi_{B}^{(2)}}, \\
m_{2}=\kappa \sigma^{2}=\frac{\left[B^{4}\right]}{\left[B^{2}\right]}=\frac{\chi_{B}^{(4)}}{\chi_{B}^{(2)}}, \\
m_{3}=\frac{\kappa \sigma}{S}=\frac{\left[B^{4}\right]}{\left[B^{3}\right]}=\frac{\chi_{B}^{(4)}}{\chi_{B}^{(3)}}
\end{gathered}
$$

Similarly we can define ratios of charge susceptibilities as well,

$$
n_{1}=\frac{\chi_{Q}^{(3)}}{\chi_{Q}^{(2)}}, n_{2}=\frac{\chi_{Q}^{(4)}}{\chi_{Q}^{(2)}}, n_{3}=\frac{\chi_{Q}^{(2)}}{\chi_{Q}^{(1)}}
$$

The computation of fluctuations and correlations of various conserved charges has formerly been done in the PNJL model for 2 flavor (Ghosh et al., 2006; Mukherjee et al., 2007) system.

\section{Result}

Higher cumulants of conserved charges serve as good scrutinisers of the location of critical point for the phase transitions under concern in the realm of relativistic heavy ion collisions. The results are correspondingly contrasted with experimental data and HRG.

Quark Number Susceptibilities : Initially, the ratios of quark number susceptibilities are studied as functions of temperature. Fig. 1 in the left panel is for zero $\mu_{q}$ whereas the right one is displayed for non-zero values of $\mu_{q}$. We get decent match with HRG results (Allton et al., 2005). For the first case, the expansion coefficients are positive for $\frac{T}{T_{c}} \leq 1$. However above $T_{c}, c_{6}$ does not remain strictly positive. This falls in line with the expectation to find chiral critical point where $c_{6}$ changes sign. Since the ratio $\frac{n_{q}}{\mu_{q} \chi_{q}}$ is connected to the isothermal compressibility, so it is expected to show a divergence for a 2 nd order phase transition. However, the absence of any such behavior in the plot signifies non-occurence of a 2 nd order phase transition.

Cumulants of Baryon Number and Charge : In Fig. 2, we displayed the variations of ratios of various orders of baryon number susceptibilities following eqn. (3) as a function of $\sqrt{s}$. It is worth mentioning that, in order to get the energy dependence of these cumulants, we have used the freeze-out parametrization done by Redlich et al. (Karsch et al., 2011). 

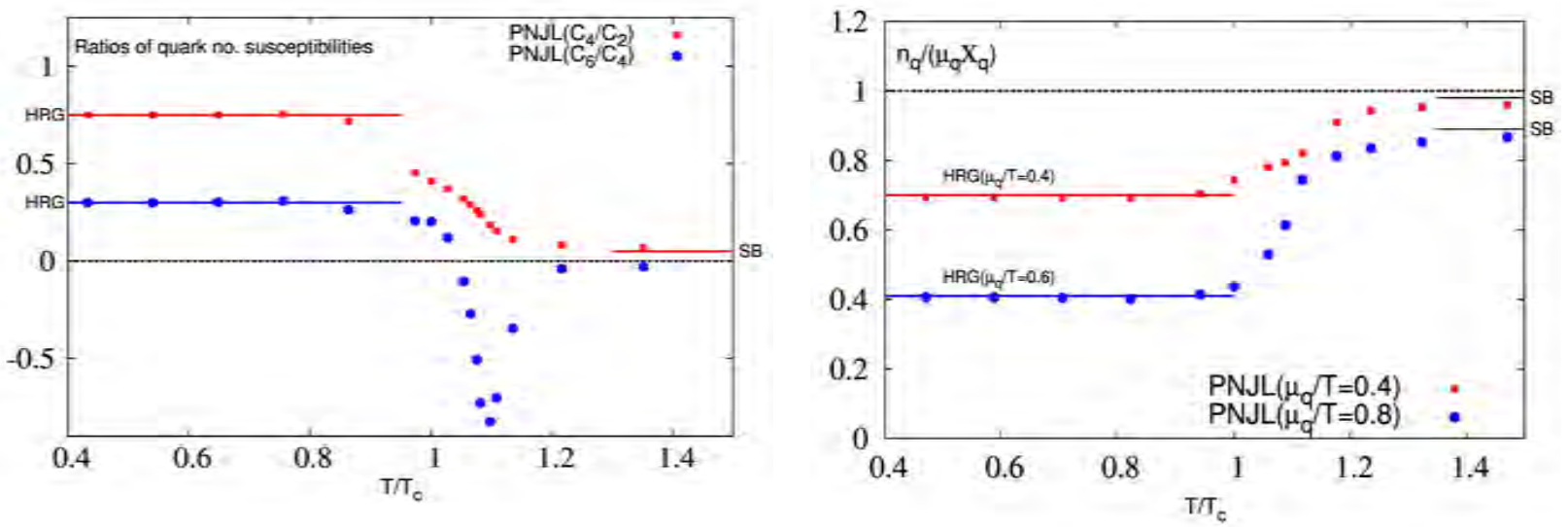

Fig. 1: Ratios of quark number susceptibilities at $0 \mu_{q}$ with $T / T_{c}$ considering 2 flavor
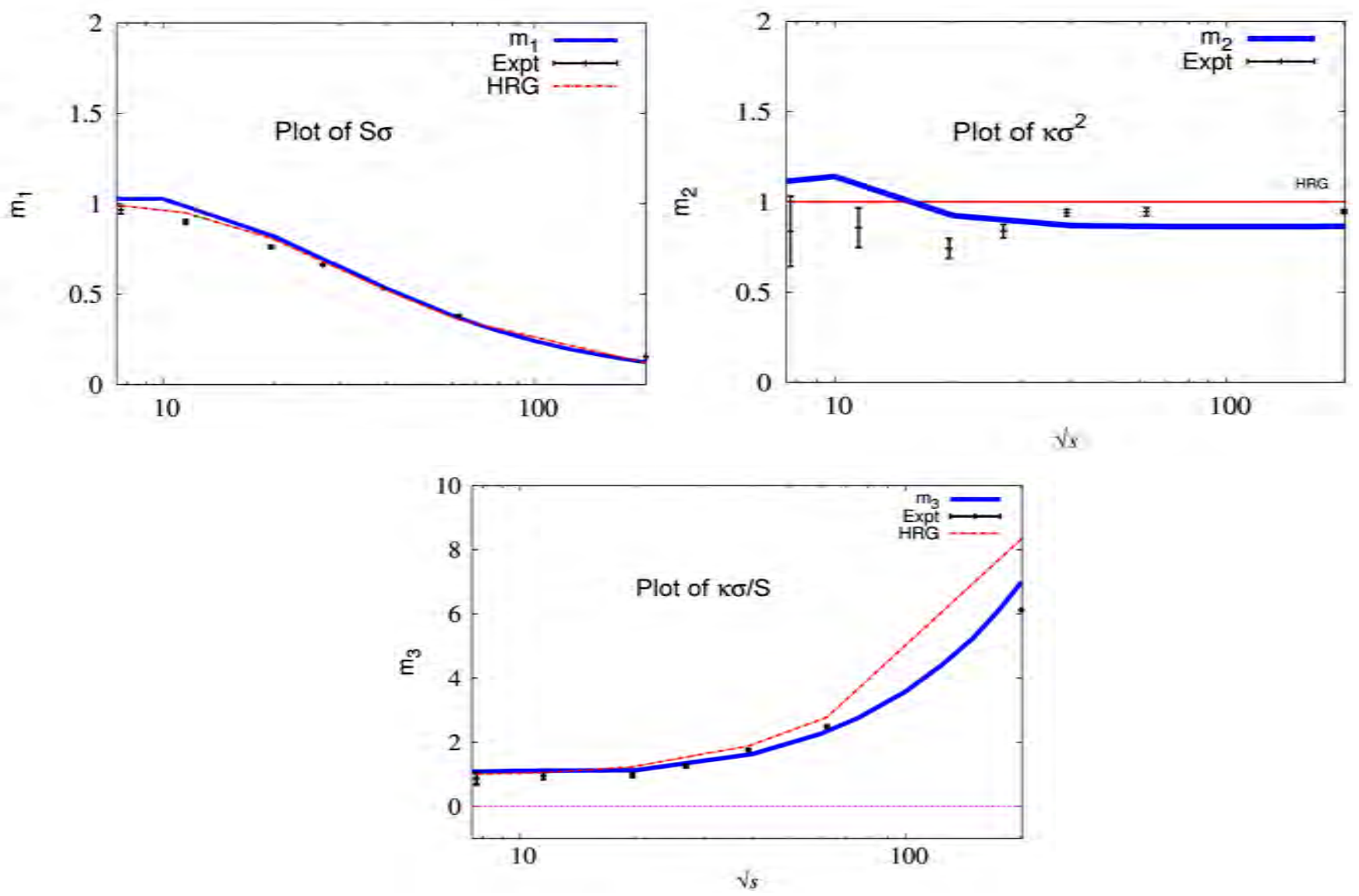

Fig. 2: Ratios of quark number susceptibilities at $0 \mu_{q}$ with $T / T_{c}$ considering 2 flavor 
As we observe here, with increase of energy $m_{1}$ is found to decrease \& $m_{3}$ increase whereas $m_{2}$ has a flatter dependence on energy. This meets the general expectations quite well. It is as well plausible to pinpoint value of the crossover temperature $T_{c}$ with unambiguous measurements of $m_{1} \& m_{3}$ at highest energies. The skewness and kurtosis are both anticipated to be positive when away from critical point, a fact well observed in our case. We have contrasted our results with that of RHIC data for $0 \%-5 \% \mathrm{Au}+\mathrm{Au}$ collisions (Adamczyk et al., 2014) alongside HRG (Karsch et al., 2011) results as well. Similarly, ratios of charge susceptibilities are plotted in Fig. 3 alongside HRG data (Karsch et al., 2011). This supposedly depending on susceptibilities and correlation length could be striking tools in the search of a possible critical point. Because of the uncertainties in size of the system produced in high-energy experiments, suitable ratios of susceptibilities are considered. However it is to be remembered that since freeze-out happens only in hadronic stage, the behavior of such ratios analysed here under different experimental conditions refer to hadronic sector and the system is in complete thermodynamic equilibrium.

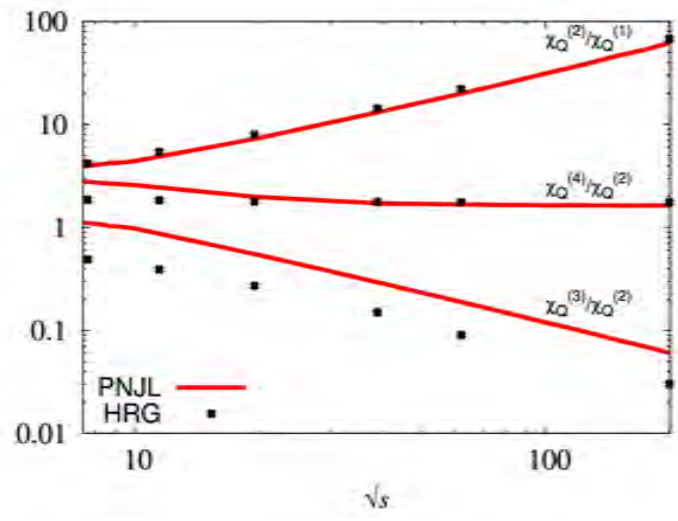

Fig. 3: Ratios of charge susceptibilities

\section{Acknowledgement}

This work is funded by CSIR, UGC (DRS \& UPE) and DST.

\section{References}

1. Adamczyk L et al., (STAR Collaboration) (2014) Energy Dependence of Moments of Net-Proton Multiplicity Distributions at RHIC, Phys Rev Lett 112032302

2. Allton CR, Dring M, Ejiri S, Hands SJ, Kaczmarek O, Karsch F, Laermann E and Redlich K Thermodynamics of two flavor QCD to sixth order in quark chemical potential, Phys Rev D71 054508

3. Bhattacharyya A, Deb P, Ghosh SK and Ray R (2010) Investigation of the phase diagram and bulk thermodynamic properties using the PolyakovNambuJona-Lasinio model with eight-quark interactions, Phys Rev D82 014021 
4. Bhattacharyya A, Deb P, Lahiri A and Ray R (2010) Susceptibililities with multiquark interactions in the Polyakov-Nambu-Jona-Lasinio model, Phys Rev D82 114028

5. Bhattacharyya A, Deb P, Lahiri A and Ray R (2011) Correlation between conserved charges in PolyakovNambu-Jona-Lasinio model with multiquark interactions, Phys Rev D83 014011

6. Bhattacharyya A, Ghosh SK, Majumder S and Ray R (2012) Study of beta equilibrated $2+1$ flavor quark matter in the Polyakov-Nambu-Jona-Lasinio model, Phys Rev D86 096006

7. Bhattacharyya A, Das S, Ghosh SK, Raha S, Ray R, Saha K and Upadhaya S (2012) Net Charge Fluctuations as a signal of QGP from Polyakov-Nambu-Jona-Lasinio model, arXiv:1212.6010 [hep-ph]

8. Gavai RV and Gupta S (2001) Quark number susceptibilities, strangeness, and dynamical confinement, Phys Rev D64 074506

9. Ghosh SK, Mukherjee TK, Mustafa MG and Ray R (2006) Susceptibilities and speed of sound from the Polyakov-Nambu-Jona-Lasinio model, Phys Rev D73 114007

10. Ghosh SK, Mukherjee TK, Mustafa MG and Ray R (2008) Polyakov-Nambu-Jona-Lasinio model with a Vandermonde term, Phys Rev D77 094204

11. Gupta S, Luo X, Mohanty B, Ritter HG and Xu N (2011) Scale for the Phase Diagram of Quantum Chromodynamics, Science 332114007

12. Jeon S and Koch V (2004) Event-by-Event Fluctuations in Quark Gluon Plasma 3 (World Scientific, Singapore), Ed. R.C. Hwa and X. N. Wang, pg. 430, arXiv:0304012 [hep-ph]

13. Karsch F and Redlich K (2011) Probing freeze-out conditions in heavy ion collisions with moments of charge fluctuations, Phys Lett $\mathbf{B 6 9 5} 136$

14. Koch V (2008) Hadronic Fluctuations and Correlations, arXiv:0810.2520v1 [nucl-th]

15. Meyer-Ortmanns H (1996) Phase transitions in quantum chromodynamics, Rev Mod Phys 68473

16. Mukherjee S, Mustafa MG and Ray R (2007) Thermodynamics of the Polyakov-Nambu-Jona-Lasinio model with nonzero baryon and isospin chemical potentials, Phys Rev D75 094015

17. Ratti C, Thaler MA and Weise W (2006) Phases of QCD: Lattice thermodynamics and a field theoretical model, Phys Rev D73 014019. 